\title{
Association between food addiction and time perspective during COVID-19 isolation
}

\author{
Mikhail F. Borisenkov ${ }^{1}$ (D) Sergey V. Popov ${ }^{1} \cdot$ Vasily V. Smirnov ${ }^{1} \cdot$ Denis G. Gubin ${ }^{2,3}$ (D) Ivan M. Petrov ${ }^{2}$. \\ Tatyana N. Vasilkova ${ }^{2}$. Svetlana V. Solovieva ${ }^{2}$. Ekaterina A. Martinson ${ }^{4}$. Anna A. Pecherkina ${ }^{5}$. Olga I. Dorogina ${ }^{5}$. \\ Elvira E. Symaniuk ${ }^{5}$
}

Received: 11 May 2021 / Accepted: 22 June 2021 / Published online: 18 February 2022

(c) The Author(s), under exclusive licence to Springer Nature Switzerland AG 2022

\begin{abstract}
Purpose The concept of time perspective (TP) implies that a mental focus on past, present, or future affect a person makes decisions and take action. Inability to plan their life for a sufficiently long time perspective due to the COVID-19 pandemic appeared to have a pronounced impact on a human's lifestyle influencing their thoughts, feelings, and behaviors, including eating behavior. This study tested two hypotheses: (a) that during COVID-19 isolation, the incidence rate of food addiction is increased, and (b) people with present TP are more likely exhibited signs of food addiction (FA).

Methods The final study sample included 949 people, mean age 21.8 7.8 years (range: $17-71$ years, women: $78.3 \%$ ). Each participant indicated their personal data and completed Yale Food Addiction Scale and the Zimbardo Time Perspective Inventory.

Results There was an increased incidence rate of FA $(\mathrm{OR}=1.678,95 \% \mathrm{CI}=1.324,2.148, p=0.000)$ during COVID-19 isolation. Individuals with balanced, future, and past positive TP were less likely to exhibit symptoms of FA. Persons with past negative, and present hedonistic TP were more likely to exhibit signs of FA.

Conclusion There was an increased incidence rate of FA during COVID-19 isolation. Persons with shortened time horizon are more likely to exhibit symptoms of FA.

Level of evidence Level V, cross-sectional descriptive study.
\end{abstract}

Keywords COVID-19 $\cdot$ Isolation $\cdot$ Food addiction $\cdot$ Time perspective

\section{Introduction}

The global spread of COVID-19 has been associated with significant changes in the lifestyle of the majority of the population. Pandemic-induced restrictions have had a significant

Mikhail F. Borisenkov

borisenkov@physiol.komisc.ru

1 Institute of Physiology of Komi Science Centre of the Ural Branch of the Russian Academy of Sciences, Syktyvkar, Russia

2 Tyumen Medical University, Tyumen, Russia

3 Tyumen Cardiology Research Centre, Tomsk National Research Medical Center, Russian Academy of Science, Tyumen, Russia

4 Vyatka State University, Kirov, Russia

5 Ural Federal University, Yekaterinburg, Russia impact on diet and food behavior. A significant increase in daily dietary energy intake during isolation was reported [1]. At the same time, there was a significant decrease in the quality of food consumed [1]. Approximately half of adults reported an increase in their intake of high-calorie, highcarbohydrate foods [2].

During the COVID-19 pandemic, limited contact between people and an associated deterioration of their psycho-emotional state have occurred. A review [3] showed a sevenfold increase in the detection rate of depression during the pandemic. Taking into account the previously noted close association between depression and food addiction (FA) [4, 5], it is expected that there will be an increase in FA detection rates during the COVID-19 pandemic. In support of this statement, it was shown that individuals who experienced distress during the COVID-19 pandemic had an increase in the number of FA symptoms [6]. 
Human's behavioral strategies and life planning may be significantly influenced as a result of economic and social instability due to COVID-19 pandemic which increases unpredictability of future. Life-history theory outlines two life-history strategies (LHS) for human adaptation to changes in the social environment: slow LHS and fast LHS [7]. Slow LHS prevails during periods of time when the social environment is stable and predictable. Under such conditions, a person has the ability to make long-term plans. Fast LHS prevails in human society when the social environment acquires features of uncertainty and unpredictability, leading to difficulty in making long-term plans. Persons with slow LHS are prone to the formation of stable family relationships [8]. Persons with fast LHS are less likely to build long-term family relationships. These people are prone to risky behavior, and neglect their health. As a consequence, they have a shorter life expectancy $[8,9]$.

Previously, the Zimbardo Time Perspective Inventory (ZTPI) [10] was shown to provide an adequate estimate of LHS. Time perspective (TP) is "the often unconscious personal attitude that each of us holds toward time and the process whereby the continual flow of existence is bundled into time categories that help to give order, coherence, and meaning to our lives" [10]. There is a close association between TP and LHS [11]. There is also a positive relationship between slow LHS and future TP, as well as between fast LHS and present TP [12]. These data indicate that the ZTPI is a useful tool for assessing the adaptive qualities of the two types of LHS during a transition from stability to uncertainty in the social environment.

Previously, numerous studies have shown that present TP is positively associated with problematic use of nicotine [13, 14], alcohol [13, 15], and drugs [13, 16], and with Internet addiction [17], while future TP is negatively correlated with these addictions. Overall, these data are consistent with the view that individuals with addiction have a shortened time horizon [18].

Although the concept of FA has numerous supporters who have now identified many similarities between FA and other types of addictions, a number of publications have expressed critical comments about the validity of introducing this term into scientific circulation [19]. Investigation of the relationship between TP and FA is of great theoretical importance. Revealing the relationship between TP and FA can be considered an additional confirmation of the validity of the FA concept (i.e., FA has similarities with nicotine, alcohol, drug and Internet addiction). Currently, there is no information about the relationship between these indicators.

The purpose of this study was, first, to compare the frequency of FA incidence before and during COVID-19 isolation and, second, to assess the relationship between TP and FA during the COVID-19 pandemic. We hypothesized that under these conditions, an increase in the frequency of FA would occur, and individuals with future TP would show a negative association with FA, whereas those with present TP would show a positive association with FA.

\section{Materials and methods}

\section{Study design}

In our previous studies $[5,20]$ conducted before COVID-19 pandemic, we analyzed the prevalence of FA in students of universities located in four cities of Russian Federation (Syktyvkar, Kirov, Yekaterinburg, and Tyumen). It was of interest to study the impact of the pandemic on this indicator. To this end, we repeatedly conducted a cross-sectional study in the same cities and universities. In addition, during COVID-19 pandemic, we analyzed the association between FA and TP.

The study was conducted free of charge, anonymously and voluntarily from April 17 to June 14, 2020 using an online questionnaire survey located on the platform Google Form. The current study was conducted according to the guidelines laid down in the Declaration of Helsinki, and all procedures involving research study participants were approved by the Ethical Committee of the Institute of Physiology, Komi Science Center the Ural Branch of the Russian Academy of Sciences (Protocol \#6, 21.03.2020). Verbal informed consent was obtained from all study participants. Verbal consent was witnessed and formally recorded.

\section{Study participants}

The invitation to participate in the survey was sent by email to teachers, researchers, postgraduates, and students of universities located in four cities in Russia. The letter contains a link to the address of the survey's website. A total of 2,500 invitations were sent. The survey was carried out until everyone who wanted to fill out the questionnaire. Inclusion criterion was as follows: informed consent to participate in the study was obtained. Questionnaires filled out with errors or omissions were excluded from the analysis. The final dataset included 949 questionnaires (response rate: 38\%). The mean age of study participants was $21.8 \pm 7.8$ years (range: 17-71 years, women: $78.3 \%$ ). The majority of the research participants were university students. During the study period, an isolation regime was introduced in Russia, requiring individuals to stay at home. Residents were allowed a limited time outside for exercise, walking pets, and visiting nearby shops and pharmacies. School and work were primarily remote; however, certain categories of persons were issued a special pass to visit their place of work and use of public transport. When asked what mode of work/ school they were participating in during the first wave of 
COVID-19 pandemic, 93\% of the study participants reported that they were in isolation. Detailed characteristics of the study participants are presented in Table 1 .

\section{Instruments}

Each participant in the study provided personal data (place of residence, sex, age, height, weight, and waist circumference) and filled out four questionnaires: Yale Food Addiction Scale (YFAS) [21], and the ZTPI [10]. Weight and height were used to calculate body mass index (BMI) as weight in kilograms divided by height in meters squared. Sex- and age-specific BMI percentiles were calculated using BMI growth charts [22]. Self-reported data on waist and height were used to calculate waist-to-height ratio (WHtR) as an indicator of abdominal obesity [23].

\section{YFAS}

The YFAS [21] was translated into Russian and used to assess FA. The scale consists of 25 questions and reveals seven diagnostic criteria for substance dependence (i.e., tolerance, withdrawal and loss of control, etc.) and clinically significant impairment related to eating behavior [21]. Two scoring options are used in the YFAS: (a) the symptom count (SC), which is equal to the sum of confirmed symptoms (range 0-7); and (b) a dichotomous measure of FA: Respondents with three or more symptoms and significant clinical impairment or distress were diagnosed with FA. Cronbach's $\alpha$ for this sample was 0.90 .

Table 1 Mean values and standard deviations of parameters measured

\begin{tabular}{lllr}
\hline Parameter, units & $\begin{array}{l}\text { Abbreviations } \\
\text { (instrument) }\end{array}$ & Mean & SD \\
\hline$N$ & & 949 & \\
$\quad$ Age, years & & 21.81 & 7.77 \\
Body mass index, $\%$ & BMI & 48.90 & 24.47 \\
Waist-to-height ratio & WHtR & 0.42 & 0.07 \\
Time perspective & TP & & \\
Past negative, scores & PA- (ZTPI) & 3.25 & 0.48 \\
Past positive, scores & PA+(ZTPI) & 2.87 & 0.70 \\
Present hedonistic, scores & PR (ZTPI) & 3.60 & 0.50 \\
Present fatalistic, scores & PR (ZTPI) & 3.59 & 0.58 \\
Future, scores & FUT (ZTPI) & 2.75 & 0.50 \\
Deviation from balanced TP, scores & DBTP (ZTPI) & 2.27 & 0.65 \\
Food addiction & & & \\
Food addiction, \% & FA (YFAS) & 15.49 & \\
Symptom counts of FA, scores & SC (YFAS) & 2.45 & 1.65 \\
\hline
\end{tabular}

ZTPI Zimbardo time perspective inventory, YFAS Yale food addiction scale

\section{ZTPI}

Time perspective was assessed using the Russian version of the ZTPI [24]. The test consists of 56 questions, divided into five subscales through which five TP options were evaluated as follows: past negative (positive) [PA-(+)], present hedonistic (fatalistic) $\left(\mathrm{PR}_{\mathrm{H}(\mathrm{F})}\right)$, and future (FUT). For this sample, Cronbach's $\alpha$ was 0.82 . We also calculated the deviation from balanced TP (DBTP) as described in [11].

\section{Statistical analyses}

The SPSS software package was used for the statistical analysis of data. To assess the impact of COVID-19 isolation on FA detection rates, the data collected in this study were compared with those of our previous studies [5, 20]. Binary logistic regression analysis was performed, in which "COVID-19 isolation" (Codes: before-0, during-1) was used as dependent variable, and sex ( 0 -females, 1 -males), age, BMI, city (1-Syktyvkar, 2-Tyumen, 3-Yekaterinburg, 4-Kirov), and FA ( 0 -no, 1-yes) were used as independent variables. A stepwise inclusion procedure was used to determine the final set of predictors in the model. Goodness of fit was evaluated by the Hosmer-Lemeshow test and Omnibus tests of model coefficients.

A series of multiple regression analyses were performed, in which PA-, PA +, PRH, PRF, FUT, and deviation from balanced TP were used as dependent variables, and the characteristics presented in Table 1 were used as independent variables (predictors). A stepwise inclusion procedure was used to determine the final set of predictors in the model. To assess multicollinearity, the variance inflation factor (VIF) was assessed. A predictor was excluded from the analysis if VIF was $\geq 5$.

Pearson's correlations were used to assess the associations among the studied indicators in males and females separately.

\section{Results}

During COVID-19 isolation, a 68\% increase in the detection rate of FA was observed (Table 2).

Individuals with past negative TP during isolation were more likely to have symptoms of FA, young age, and signs of visceral obesity (Table 3: model 1). These predictors explained $11.4 \%$ of the variability in the past negative TP.

Past positive TP was found to be more common in females (Table 3: model 2). This predictor explained $1.1 \%$ of the variability in the past positive TP. 
Table 2 Results of logistic regression analysis

\begin{tabular}{|c|c|c|c|c|c|c|c|c|c|}
\hline \multirow[t]{2}{*}{ Dependent variable } & \multirow[t]{2}{*}{ Predictors } & \multirow[t]{2}{*}{$B$} & \multirow[t]{2}{*}{ OR } & \multirow[t]{2}{*}{$95 \% \mathrm{CI}$} & \multirow[t]{2}{*}{$P^{\&}$} & \multicolumn{2}{|c|}{ Omnibus test } & \multicolumn{2}{|c|}{$\begin{array}{l}\text { Hosmer- } \\
\text { Lemeshow } \\
\text { test }\end{array}$} \\
\hline & & & & & & $\overline{\chi^{2}}$ & $P$ & $\overline{\chi^{2}}$ & $P$ \\
\hline \multirow[t]{3}{*}{ COVID-19 isolation } & Sex & -0.430 & 0.645 & $0.533-0.779$ & 0.000 & 272.196 & 0.000 & 4.369 & 0.822 \\
\hline & Age & 0.109 & 1.115 & $1.096-1.135$ & 0.000 & & & & \\
\hline & FA & 0.523 & 1.687 & $1.324-2.148$ & 0.000 & & & & \\
\hline
\end{tabular}

$F A$ food addiction, $B$ non-standardized regression coefficient, $O R$ odds ratio, $C I$ confidence interval
Table 3 Results of multiple regression analyses

\begin{tabular}{|c|c|c|c|c|c|c|c|c|}
\hline Model \# & Dependent variable & Predictors & $B$ & $\beta$ & $P$ & $R^{2}$ & $\Delta R^{2}$ & VIF \\
\hline \multirow[t]{3}{*}{1} & \multirow[t]{3}{*}{ PA- } & $\mathrm{SC}$ & 0.123 & 0.289 & 0.000 & 0.096 & 0.096 & 1.024 \\
\hline & & Age & -0.011 & -0.131 & 0.000 & 0.108 & 0.012 & 1.078 \\
\hline & & WHtR & 0.799 & 0.076 & 0.039 & 0.114 & 0.006 & 1.073 \\
\hline 2 & $\mathrm{PA}+$ & Sex & -0.153 & -0.103 & 0.006 & 0.011 & 0.011 & 1.000 \\
\hline \multirow[t]{3}{*}{3} & \multirow[t]{3}{*}{$\mathrm{PR}_{\mathrm{H}}$} & $\mathrm{SC}$ & 0.068 & 0.230 & 0.000 & 0.066 & 0.066 & 1.015 \\
\hline & & Age & -0.012 & -0.203 & 0.000 & 0.110 & 0.044 & 1.017 \\
\hline & & Sex & -0.113 & -0.089 & 0.013 & 0.117 & 0.007 & 1.010 \\
\hline 4 & $\mathrm{PR}_{\mathrm{F}}$ & $\mathrm{SC}$ & 0.063 & 0.213 & 0.000 & 0.046 & 0.046 & 1.000 \\
\hline \multirow[t]{2}{*}{5} & \multirow[t]{2}{*}{ FUT } & $\mathrm{SC}$ & -0.051 & -0.169 & 0.000 & 0.026 & 0.026 & 1.005 \\
\hline & & Sex & -0.144 & -0.111 & 0.003 & 0.038 & 0.012 & 1.005 \\
\hline \multirow[t]{2}{*}{6} & \multirow[t]{2}{*}{$\mathrm{DBTP}^{1}$} & $\mathrm{SC}$ & 0.098 & 0.251 & 0.000 & 0.060 & 0.060 & 1.005 \\
\hline & & Sex & 0.139 & 0.083 & 0.023 & 0.067 & 0.007 & 1.005 \\
\hline
\end{tabular}

$P A+(-)$ past positive (negative) time perspective (TP), $P R_{H(F)}$ present hedonistic (fatalistic) TP, FUT future TP, DBTP deviation from balanced TP, WHtR waist-to-height ratio, SC symptom counts of food addiction. A series of multiple regression analyses were performed using $\mathrm{PA}+(-), \mathrm{PR}_{\mathrm{H}(\mathrm{F})}$, FUT, and DBTP as dependent variables and Age, Sex (0-female; 1 -male), City (1-Syktyvkar; 2-Tyumen; 3 Yekaterinburg; 4-Kirov), BMI, WHtR, and SC as independent variables (predictors); a stepwise inclusion procedure was used to determine the final set of predictors in the model. To assess multicollinearity, the variance inflation factor (VIF) was assessed. $B=$ non-standardized regression coefficient; $\beta=$ standardized regression coefficient; $P=$ significance of regression coefficient; $R^{2}=$ total variance accounted by predictors at their stepwise inclusion in the model; $\Delta R^{2}=$ portion of the variance accounted for by separate predictors in the model; ${ }^{1}$ in this model, the DBTP was evaluated. Therefore, interpretation of the signs of the regression coefficients was opposite to that used in models $1-5$
Present hedonistic TP was more likely to have symptoms of FA, young age, and more common in females (Table 3: model 3). These predictors explained $11.7 \%$ of the variability in the present hedonistic TP.

Present fatalistic TP was more likely to exhibit symptoms of FA (Table 3, model 4). This predictor explained $4.6 \%$ of the variability in present fatalistic TP.

Future TP was more common in females. People with this TP are less likely to have symptoms of FA (Table 3: model 5). These predictors explained $3.8 \%$ of the variability in future TP.

Balanced TP was more common in females. People with this TP are less likely to have symptoms of FA (Table 3: model 6 ). These predictors explained $6.7 \%$ of the variability in balanced TP.

The correlation analysis showed that the nature of the associations among the TPs and SC is the same in males
Table 4 Results of correlation analyses 
and females (Table 4). There are only slightly higher correlation coefficients in females compared to males, which may be due to the fact that the sample is not balanced by sex.

\section{Discussion}

We have shown that there was a significant increase in the detection rate of FA during the isolation caused by the COVID-19 pandemic. These findings are consistent with the results of other authors [6] who showed that individuals who experienced COVID-19-induced distress during the pandemic experienced an increase in the number of FA symptoms.

To our knowledge this is the first study to explore the association between TP and FA. We showed that individuals with balanced and future TP were less likely to have FA symptoms, whereas individuals with present hedonistic, present fatalistic, and past negative TP were more often to have FA.

Previous work has shown significant associations between TP and drug [16], alcohol [15], and Internet [25] addiction. A negative association has been found between future TP and all three types of dependencies [15, 16, 25]. Alcohol addiction was also found to be negatively associated with past positive TP. In addition, a positive association was noted between Internet addiction and present TP [25], alcohol addiction, and present hedonistic, present fatalistic, and past negative TP [15]. In general, these data are consistent with our results and indicate that FA, as well as other types of dependencies, may be characteristic of individuals with a short time horizon [18]. Our data on the nature of the relationship between FA and TP indicate similarities between FA and other types of dependencies, thus providing additional confirmation of the validity of the FA concept.

The increase in the frequency of FA incidence during the COVID-19 pandemic is consistent with the data on increased incidence rate of other types of addictions under these conditions, for example, Internet addiction [26, 27], alcohol [28], and substance use disorders [26]. There was also a threefold increase in the frequency of detection of symptoms of depression during the COVID-19 pandemic [29]. The deterioration of the psycho-emotional state observed during the COVID-19 pandemic may be one of the reasons for the increase in the incidence rate of addictive disorders in persons with present TP. Recently, there have been data, indicating that in some pathologies (for example, PTSD), it can be effectively corrected using a complex of psychotherapeutic treatments called TP therapy [30]. Therefore, TP therapy can be effective in preventing the negative effects of prolonged isolation of persons with present TP.

\section{Strengths and limitations}

There are several limitations to this study; first, an imbalanced sample in terms of the sex and age of the study participants. In addition, a large percentage of people declined to participate in the study, most likely due to a high level of existing stress due to the pandemic. Therefore, the study may not accurately represent the total population due to bias in who responded. Finally, this study used a cross-sectional design, so we cannot evaluate causal relationships between the studied indicators.

\section{Conclusions}

In this work, we showed that there is a significant increase in incidence rate of FA during the COVID-19 isolation. The association between TP and FA was studied for the first time. During the first wave of the pandemic, higher numbers of FA symptoms were observed in individuals with past negative, present hedonistic, and present fatalistic TP. The lowest numbers of FA symptoms were observed in individuals with balanced and future TP.

\section{What is already known on this subject?}

Previous investigations indicate that human's time perspective is a strong indicator of psychological wellbeing and some psychological disorders. It was shown that shortened time horizon in human is associated with increased risk of addictive disorders such as alcohol, drug, and Internet addiction.

\section{What your study adds?}

A significant association between time perspective and food addiction was noted for the first time. Present fatalistic time perspective was shown to be associated with increased incidence rate of food addiction. At the same time, balanced and future time perspective are associated with low incidence rate of food addiction. These data confirm the validity of the concept of food addiction and also serve as a scientific basis for using time perspective therapy for the prevention of food addiction.

Funding No Grant was obtained for this research.

\section{Declarations}

Conflict of interest The authors declare no conflict of interest.

Ethical approval This study was approved by the ethics committee of the Institute of Physiology of Komi Science Center, Ural Branch of 
RAS, and was conducted in accordance with the ethical standards of the Helsinki Declaration.

Informed consent Informed consent was obtained from all study participants.

\section{References}

1. Marty L, de Lauzon-Guillain B, Labesse M, Nicklaus S (2020) Food choice motives and the nutritional quality of diet during the COVID-19 lockdown in France. Appetite 157:105005. https://doi. org/10.1016/j.appet.2020.105005

2. Buckland NJ, Swinnerton LF, Ng K, Price M, Wilkinson LL, Myers A, Dalton M (2021) Susceptibility to increased high energy dense sweet and savoury food intake in response to the COVID19 lockdown: the role of craving control and acceptance coping strategies. Appetite 158:105017. https://doi.org/10.1016/j.appet. 2020.105017

3. Bueno-Notivol J, Gracia-García P, Olaya B, Lasheras I, LópezAntón R, Santabárbara J (2021) Prevalence of depression during the COVID-19 outbreak: a meta-analysis of community-based studies. Int J Clin Health Psychol 21(1):100196. https://doi.org/ 10.1016/j.ijchp.2020.07.007

4. Burrows T, Kay-Lambkin F, Pursey K, Skinner J, Dayas C (2018) Food addiction and associations with mental health symptoms: a systematic review with meta-analysis. J Human Nutr Diet 31(4):544-572. https://doi.org/10.1111/jhn.12532

5. Borisenkov MF, Tserne TA, Bakutova LA (2018) Food addiction in Russian adolescents: associations with age, sex, weight, and depression. Eur Eat Disord Rev 26(6):671-676. https://doi.org/ 10.1002/erv.2644

6. Panno A, Carbone GA, Massullo C, Farina B, Imperatori C (2020) COVID-19 Related distress is associated with alcohol problems, social media and food addiction symptoms: insights from the Italian experience during the lockdown. Front Psychiatry 11:1314. https://doi.org/10.3389/fpsyt.2020.577135

7. Figueredo AJ, de Baca TC, Woodley MA (2013) The measurement of human life history strategy. Personality Individ Differ 55:251-255. https://doi.org/10.1016/j.paid.2012.04.033

8. Figueredo AJ, Vasquez G, Brumbach BH, Schneider SM, Sefcek JA, Tal IR, Jacobs W (2006) Consilience and life history theory: from genes to brain to reproductive strategy. Dev Rev 26:243-275. https://doi.org/10.1016/j.dr.2006.02.002

9. Ellis BJ, Figueredo AJ, Schlomer GL (2009) Fundamental dimensions of environmental risk: the impact of harsh versus unpredictable environments on the evolution and development of life history strategies. Hum Nature 20:204-268. https://doi.org/10.1007/ s12110-009-9063-7

10. Zimbardo PG, Boyd JN (1999) Putting time in perspective: a valid, reliable individual-differences metric. J Pers Soc Psychol 77:1271-1288. https://doi.org/10.1037/0022-3514.77.6.1271

11. Stolarski M, Fieulaine N, Zimbardo PG (2018) Putting time in a wider perspective: the past, the present, and the future of time perspective theory. In: Zeigler-Hill V, Shackelford T (eds) The SAGE handbook of personality and individual differences. SAGE, Thousand Oakes, pp 592-628

12. Stolarski M, Czarna AZ, Malesza M, Szymańska A (2017) Here and now: sociosexuality mediates the associations between dark triad and time perspectives (in females). Personality Individ Differ 111:119-123. https://doi.org/10.1016/j.paid.2017.02.001

13. Keough KA, Zimbardo PG, Boyd JN (1999) Who's smoking, drinking, and using drugs? Time perspective as a predictor of substance use. Basic Appl Soc Psychol 21(2):149-164. https:// doi.org/10.1207/S15324834BA210207

14. Sansone G, Fong GT, Hall PA, Guignard R, Beck F, Mons U et al (2013) Time perspective as a predictor of smoking status: findings from the International Tobacco Control (ITC) Surveys in Scotland, France, Germany, China, and Malaysia. BMC Public Health 13(1):346-354. https://doi.org/10.1186/ 1471-2458-13-346

15. Loose TT, Acier D, Andretta JR, Cole JC, McKay MT, Wagner V, Worrell FC (2018) Time perspective and alcohol-use indicators in France and the United Kingdom: results across adolescents, university students, and treatment outpatients. Addict Res Theory 26(2):143-150. https://doi.org/10.1080/16066359.2017.1334202

16. Apostolidis T, Fieulaine N, Simonin L, Rolland G (2006) Cannabis use, time perspective and risk perception: evidence of a moderating effect. Psychol Health 21(5):571-592. https://doi.org/ $10.1080 / 14768320500422683$

17. Chittaro L, Vianello A (2013) Time perspective as a predictor of problematic internet use: a study of Facebook users. Personality Individ Differ 55(8):989-993. https://doi.org/10.1016/j.paid.2013. 08.007

18. Petry NM, Bickel WK, Arnett M (1998) Shortened time horizons and insensitivity to future consequences in heroin addicts. Addiction 93(5):729-738. https://doi.org/10.1046/j.1360-0443. 1998.9357298.x

19. Ziauddeen H, Farooqi IS, Fletcher PC (2012) Obesity and the brain: how convincing is the addiction model? Nat Rev Neurosci 13(4):279-286. https://doi.org/10.1038/nrn3212

20. Borisenkov MF, Popov SV, Pecherkina AA, Dorogina OI, Martinson EA, Vetosheva VI et al (2020) Food addiction in young adult residents of Russia: associations with emotional and anthropometric characteristics. Eur Eat Disord Rev 28(4):465-472. https://doi. org/10.1002/erv.2731

21. Gearhardt AN, Corbin WR, Brownell KD (2009) Preliminary validation of the Yale food addiction scale. Appetite 52:430-436. https://doi.org/10.1016/j.appet.2008.12.003

22. World Health Organization (2007) Growth reference data for 5-19 years. https://www.who.int/tools/growth-refer ence-data-for-5to19-years

23. Swainson MG, Batterham AM, Tsakirides C, Rutherford ZH, Hind K (2017) Prediction of whole-body fat percentage and visceral adipose tissue mass from five anthropometric variables. PLoS ONE 12:e0177175. https://doi.org/10.1371/journal.pone.0177175

24. Sircova A, Sokolova ET, Mitina OV (2008) Adaptation of Zimbardo time perspective inventory. Psychol J (Psikhologicheskii Zhurnal) 29:101-109 ((In Russian))

25. Kim J, Hong H, Lee J, Hyun MH (2017) Effects of time perspective and self-control on procrastination and Internet addiction. J Behav Addict 6:229-236. https://doi.org/10.1556/2006.6.2017. 017

26. Sun Y, Li Y, Bao Y, Meng S, Sun Y, Schumann G et al (2020) Brief report: increased addictive internet and substance use behavior during the COVID-19 pandemic in China. Am J Addict 29(4):268-270. https://doi.org/10.1111/ajan.13066

27. Dong H, Yang F, Lu X, Hao W (2020) Internet addiction and related psychological factors among children and adolescents in china during the coronavirus disease 2019 (COVID-19) epidemic. Front Psychiatry 11:00751. https://doi.org/10.3389/fpsyt.2020. 00751

28. Ahmed MZ, Ahmed O, Aibao Z, Hanbin S, Siyu L, Ahmad A (2020) Epidemic of COVID-19 in China and associated psychological problems. Asian J Psychiatry 51:102092. https://doi.org/ 10.1016/j.ajp.2020.102092

29. Ettman CK, Abdalla SM, Cohen GH, Sampson L, Vivier PM, Galea S (2020) Prevalence of depression symptoms in US adults before and during the COVID-19 pandemic. JAMA Netw Open 
3(9):e2019686. https://doi.org/10.1001/jamanetworkopen.2020. 19686

30. Sword RM, Sword RKM, Brunskill SR, Zimbardo PG (2014) Time perspective therapy: a new time-based metaphor therapy for PTSD. J Loss \& Trauma 19(3):197-201. https://doi.org/10. $1080 / 15325024.2013 .763632$
Publisher's Note Springer Nature remains neutral with regard to jurisdictional claims in published maps and institutional affiliations. 